\title{
Amplification of the androgen receptor may not explain the development of androgen-independent prostate cancer
}

\author{
J. EDWARDS, N.S. KRISHNA, R. MUKHERJEE, A.D. WATTERS, M.A. UNDERWOOD* \\ and J.M.S. BARTLETT \\ University Departments of Surgery and *Urology, Glasgow Royal Infirmary, Glasgow, Scotland, UK
}

Objective To examine the role of androgen receptor (AR) gene amplification and aneusomy of the X chromosome in the development of antiandrogen-resistant prostate cancer.

Patients and methods Twenty patients with prostate cancer resistant to androgen-deprivation therapy were selected for study. The records of patients with tumours before and after antiandrogen therapy, and with a full clinical follow-up, were retrieved. AR gene amplification and $\mathrm{X}$ chromosome copy number were assessed by fluorescence in situ hybridization using a labelled probe at locus Xq11-13 for the AR gene and a labelled $\alpha$-satellite probe for the $\mathrm{X}$ chromosome. At least 20 nuclei were scored over three tumour areas by two independent observers.

Results Aneusomy of the $\mathrm{X}$ chromosome was reported respectively in seven (35\%) and 11 (55\%) tumours before and after hormone relapse, the AR gene copy number was increased in seven (35\%) and 13 (65\%), respectively, and AR gene amplification was detected in one $(5 \%)$ and three $(15 \%)$, respectively. Neither increased AR copy number nor AR amplification in primary tumours precluded a biological response to androgen-deprivation therapy.

Conclusion The rate of AR gene amplification is too low to be solely responsible for the development of antiandrogen-resistant prostate cancer. Also, the presence of amplified AR and cells aneusomic for the $\mathrm{X}$ chromosome in primary tumours that respond to androgen-deprivation therapy suggests that an increase in AR gene copy number does not prevent a tumour from responding to this therapy. Therefore other mechanisms which could cause hormonerefractory prostate cancer must be investigated before it is understood why so many patients relapse with this disease.

Keywords prostate cancer, androgen receptor, X chromosome, fluorescence in situ hybridization

\section{Introduction}

The incidence of prostate cancer is higher in men from the western world than any other cancer and is the second most frequent cause of male cancer-related deaths [1-3]. As androgens regulate normal growth and differentiation of the prostate gland, it is not surprising that prostate cancer growth is stimulated by androgens [4]. The effects of androgens on prostate tissue can be inhibited by androgen receptor (AR) antagonists (antiandrogens) [5]; $70-80 \%$ of men with prostate cancer treated with antiandrogens respond favourably [6]. However, this effect is transient [7], with most patients eventually developing androgen-resistant disease [8].

Several theories, e.g. mutation of the AR [7], clonal expansion of tumour cells hypersensitive to androgens [9] and increased expression of AR [4], have been proposed as a possible explanation as to why prostate cancer develops resistance to antiandrogens or

Accepted for publication 6 June 2001 androgen-deprivation therapy. Koivisto et al. [10] postulated that amplification of the AR gene plays a key role in progression to refractory prostate cancer $[9,10]$. They reported that six of 10 recurrent prostate tumours resistant to antiandrogens and androgendeprivation therapy were polysomic for the X chromosome and that three had amplification of the AR gene [10]. They hypothesized that the combination of these factors caused the development of prostate cancer resistant to current first-line therapy, i.e. an increase in AR protein levels would result in proliferative advantages during antiandrogen therapy [10]. Further studies with more patients confirmed that polysomy of $\mathrm{X}$ chromosome ranges is detectable in $42-60 \%$ of relapsed tumours from patients with prostate cancer, and amplification of the AR gene has been reported in $20-30 \%$ of recurrent tumours from the same patient cohorts [9-12].

The largest study to date (339 samples) reported no AR amplifications in any of the 223 primary tumours investigated [12]. However, there is still no clear 
investigation into AR gene amplification before hormonal therapy and after the development of antiandrogen resistance (after hormonal therapy) using paired tumours from the same patient. In the present pilot study we investigated AR gene amplification and $\mathrm{X}$ chromosome status in 42 tumours from 20 patients, all with paired primary and relapsed (after androgen-deprivation therapy) tumours from the same patient.

\section{Patients and methods}

Twenty patients (mean age at diagnosis 71.1 years, range 55-83) were retrospectively selected for analysis; ethical approval was obtained from the local research and ethical committee for the use of their tissue in the study. All patients received conventional androgendeprivation therapy (orchidectomy or antiandrogens). Patients were selected for analysis if they initially responded to treatment (the response being defined by the PSA level decreasing by at least half) but subsequently relapsed. PSA values and a full clinical follow-up were available for each patient. Patients were classed as having hormone-refractory cancer when sustained rising PSA levels were recorded and they were selected for study if a tumour sample was available after hormone relapse. The initial tumour sample was either from TURP or TRUS-guided biopsy, but the relapsed tumour sample was always from TURP, which was used to treat clinical symptoms.

Sections $(5 \mu \mathrm{m})$ were cut from archival formalin-fixed, paraffin-embedded tissue and placed on aminopropyl triethoxysilane-treated slides. The slides were pretreated on a VP2000 robotic slide processor (Vysis Ltd, Surrey, UK). This involved dewaxing and rehydration, pretreatment with $8 \%$ sodium thiosulphate at $80^{\circ} \mathrm{C}$ for $30 \mathrm{~min}$ and digestion with $0.05 \%$ pepsin at $37^{\circ} \mathrm{C}$ for $26 \mathrm{~min}$. Tissue sections were subsequently post-fixed in 10\% formalin for $10 \mathrm{~min}$ and dehydrated through increasing concentrations of ethanol.

Slides were denatured at $72^{\circ} \mathrm{C}$ for $2 \mathrm{~min}$; dual-labelling hybridization with CEP buffer (Vysis) containing X chromosome $\alpha$-satellite probe (Spectrum Green ${ }^{\circledR}$ labelled CEP X, Vysis) and AR probe (Spectrum Orange ${ }^{\circledR}$ labelled probe locus Xq11-13, Vysis) was undertaken at $37^{\circ} \mathrm{C}$ overnight on a Misha unit (Shandon, Runcorn, UK). Slides were then washed in $0.4 \times$ saline-citrate buffer, $0.3 \% \mathrm{NP}-40$ at $72^{\circ} \mathrm{C}$ for $2 \mathrm{~min}$, allowed to air dry and mounted in $0.5 \mu \mathrm{g} / \mathrm{mL}$ 4,6-diamindino-2 phenylindole-2 hydrochloride in Vectashield antifade (Vetrolabs, Peterborough, UK). Signals were visualized using a microscope with a $100 \mathrm{~W}$ mercury lamp, with a triple band-pass filter block specific for the excitation and emission wavelengths of the Spectrum Green and Spectrum Orange fluors (Vysis).
Serially sectioned haematoxylin and eosin-stained tissue sections were first examined microscopically to locate the tumour areas. Sections stained for fluorescence in situ hybridization were then scanned at $\times 100$ magnification to locate the tumour areas. Twenty nonoverlapping nuclei per section were evaluated from three different areas by two independent observers, using a $\times 100$ objective. Signals per nucleus for X chromosome (green) and AR (orange) were counted on a cell-by-cell basis and the results recorded manually; cells with no signals for either $\mathrm{X}$ or $\mathrm{AR}$ were ignored. The mean chromosomal copy number for the $\mathrm{X}$ chromosome was calculated by totalling the number of green signals (X probe) counted in a specific area and dividing by the number of nuclei assessed. Similarly, the mean gene copy number of the AR was calculated by totalling the number of orange signals (AR probe) counted in a specific area and dividing this by the number of nuclei assessed. Analysis of copy number in thin tissue sections can be affected by nuclear truncation $[12,13]$ and we excluded nuclei with missing signals to ensure that the gene:chromosome ratio was more accurately represented. Therefore, the normal range for $\mathrm{X}$ chromosome and AR copy number were identified using the mean chromosomal copy number from $14 \mathrm{BPH}$ (as a genetically stable control) samples \pm 3 SD $(\approx 99 \%$ CI $)$. Using this approach the normal range for the $\mathrm{AR}$ and chromosome $\mathrm{X}$ were $0.91-1.30$ and 0.97-1.23, respectively. The AR:X chromosome ratio in $14 \mathrm{BPH}$ samples was $0.93-1.07$ $(1.04 \pm 3 \mathrm{sD})$. Amplification was defined as an AR : X ratio of $>1.5$ [9]. If tumours were heterogeneous the area with most abnormalities was used in the final analysis.

Fisher's exact test was used to compare the level of $\mathrm{X}$ chromosome copy number, AR copy number and AR gene amplification between primary tumours and those after hormone deprivation therapy.

\section{Results}

Of the 20 patients, two had bone metastases at the time of initial hormone therapy; 42 tumours were analysed, including 20 primary tumours and 20 tumours after relapse; in addition, two patients underwent TURP during their PSA response and these samples were also analysed. Of the 20 primary tumours, $11(55 \%)$ had a Gleason sum of $\leqslant 6$, four $(20 \%)$ a sum of 7 and three $(15 \%)$ a sum of $\geqslant 8$. The Gleason score was unavailable for two primary tumours. The Gleason sum for the relapsed tumour was always the same or higher than that of the primary tumour; two patients had a Gleason sum of $7(10 \%)$ in the relapsed tumour and $18(90 \%)$ had a Gleason score of $\geqslant 8$. One tumour (after hormone relapse) showed heterogeneity (2.3\%). In six cases no abnormalities of either the X chromosome or AR copy 
number were detected in either the biopsies taken before or after hormone relapse.

Seven $(35 \%)$ of the primary tumours and $11(55 \%)$ relapsed tumours were polysomic for the $\mathrm{X}$ chromosome (Table 1). There was no significant difference in the number of tumours with aneusomy $\mathrm{X}$ before and after hormone relapse $(P=0.2)$. In two cases the primary tumours were aneusomic for chromosome $\mathrm{X}$ and normosomic in the relapsed tumour.

Seven (35\%) tumours before hormone relapse and 13 $(65 \%)$ after $(P=0.11)$ had increased copies of the AR (Table 1). In one case the primary tumour had increased copies of AR, but only one copy in the relapsed tumour.

Amplification of the AR gene was detected in one (5\%) primary and three $(15 \%)$ hormone-relapsed tumours (Table 1); there was therefore no significant difference in the level of AR gene amplification when primary and hormone-relapsed tumours were compared $(P=0.6)$. There was no association between the Gleason sum in the primary or relapsed tumour and AR amplification. From the three patients with amplified AR in the hormonerelapsed tumour, one primary tumour had a Gleason sum of 9 , two a Gleason sum of $\leqslant 6$, and two relapsed tumours had a Gleason sum of $\geqslant 8$ and one a Gleason sum of 7. A further two hormone-relapsed tumours had high AR:X ratios (1.39 and 1.41), but did not appear to be amplified (ratio <1.5). From the patients with amplified hormone-relapsed tumours, two had undergone orchidectomy and three had received antiandrogen therapy. Therefore, the type of treatment received did not appear to influence AR amplification.
Serial PSA levels were used to document the hormonal response and relapse in all patients (Fig. 1). In patients where there were no AR abnormalities down-regulation of PSA by hormonal therapy and subsequent PSA escape occurred as expected (e.g. Fig. 1a). In the case illustrated the biopsy after relapse was obtained following a 270-fold rise in PSA over 14 months (Fig. 1a). In the one case with amplification of AR in biopsies both before and after relapse (Fig. 1b) the PSA level rose from $0.9 \mu \mathrm{g} / \mathrm{L}$ to $1450 \mu \mathrm{g} / \mathrm{L}$ during hormone escape, with no increase in amplification of AR. Finally, the PSA response in one of two cases with amplification of $A R$ in the hormone-relapse biopsy only is shown in Fig. 1c.

\section{Discussion}

Hormone-refractory prostate cancer has been postulated to develop because of amplification of the AR gene [9]. Several studies supporting this hypothesis investigated levels of AR gene amplifications and aneusomy of chromosome $\mathrm{X}$ in recurrent tumours from patients who initially responded to androgen-deprivation therapy $[4,9,11,12]$. Visakorpi et al. [9] reported that $30 \%$ of recurrent tumour specimens resistant to androgendeprivation therapy had AR gene amplification. Although this level of amplification was too low to be the sole cause of hormone-refractory prostate cancer, it was suggested that an increase in AR copy number from either AR amplification or duplication of the X chromosome might be responsible. Visakorpi et al. [9] reported that a further $35 \%$ of tumours investigated were

Table 1 Cases with abnormal X chromosome or AR gene copy number, or an abnormal AR:X chromosome copy number ratio

\begin{tabular}{|c|c|c|c|c|c|c|}
\hline \multirow[b]{2}{*}{ Patient no. } & \multicolumn{2}{|c|}{ X chromosome, biopsy } & \multicolumn{2}{|l|}{$A R$, biopsy } & \multicolumn{2}{|l|}{$A R: X$ ratio } \\
\hline & before (mid) & after relapse & before (mid) & after relapse & before (mid) & after relapse \\
\hline 2 & 1.63 & 1.58 & 1.61 & 1.63 & & \\
\hline 3 & $1.17(1.06)$ & 1.41 & $1.05(1.07)$ & 1.33 & & \\
\hline 5 & 1.32 & 1.46 & 1.42 & 1.48 & & \\
\hline 6 & 1.03 & 1.68 & 1.05 & 2.33 & 1.02 & 1.39 \\
\hline 7 & $2.99(2.72)$ & 1.5 & $8.68(11.87)$ & 4.63 & $2.90(4.36)$ & 3.09 \\
\hline 8 & 1.03 & 1.61 & 1.03 & 1.61 & & \\
\hline 9 & 1.46 & 1.04 & 1.48 & 1.04 & & \\
\hline 10 & 1.13 & 1.58 & 1.1 & 1.48 & & \\
\hline 13 & 1.07 & 1.53 & 1.08 & 2.15 & 1.01 & 1.41 \\
\hline 16 & 1.22 & 1.79 & 1.22 & 1.85 & & \\
\hline 17 & 1.48 & 1.43 & 1.54 & 1.53 & & \\
\hline 18 & 1.17 & 1.25 & 1.2 & 1.32 & & \\
\hline 19 & 1.38 & 1.1 & 1.46 & 2.65 & 1.06 & 2.45 \\
\hline 20 & 1.34 & 1.35 & 1.31 & 6.38 & 1.00 & 4.90 \\
\hline
\end{tabular}

The mean X chromosome copy number per nucleus is given for each case if either of the biopsies before or after relapse had a mean chromosomal copy number of $\geqslant 1.3$. The mean AR gene copy number per nucleus is given for each case if either of the biopsies before or after relapse had a mean gene copy number of $\geqslant 1.23$. The mean $\mathrm{AR}: \mathrm{X}$ chromosome ratio is given for each case that had an $\mathrm{AR}: \mathrm{X}$ chromosome ratio of $\geqslant 1.07$, as above this value is considered abnormal. Cases were considered as having AR amplification if their mean AR:X chromosome ratio was $>1.5$. 

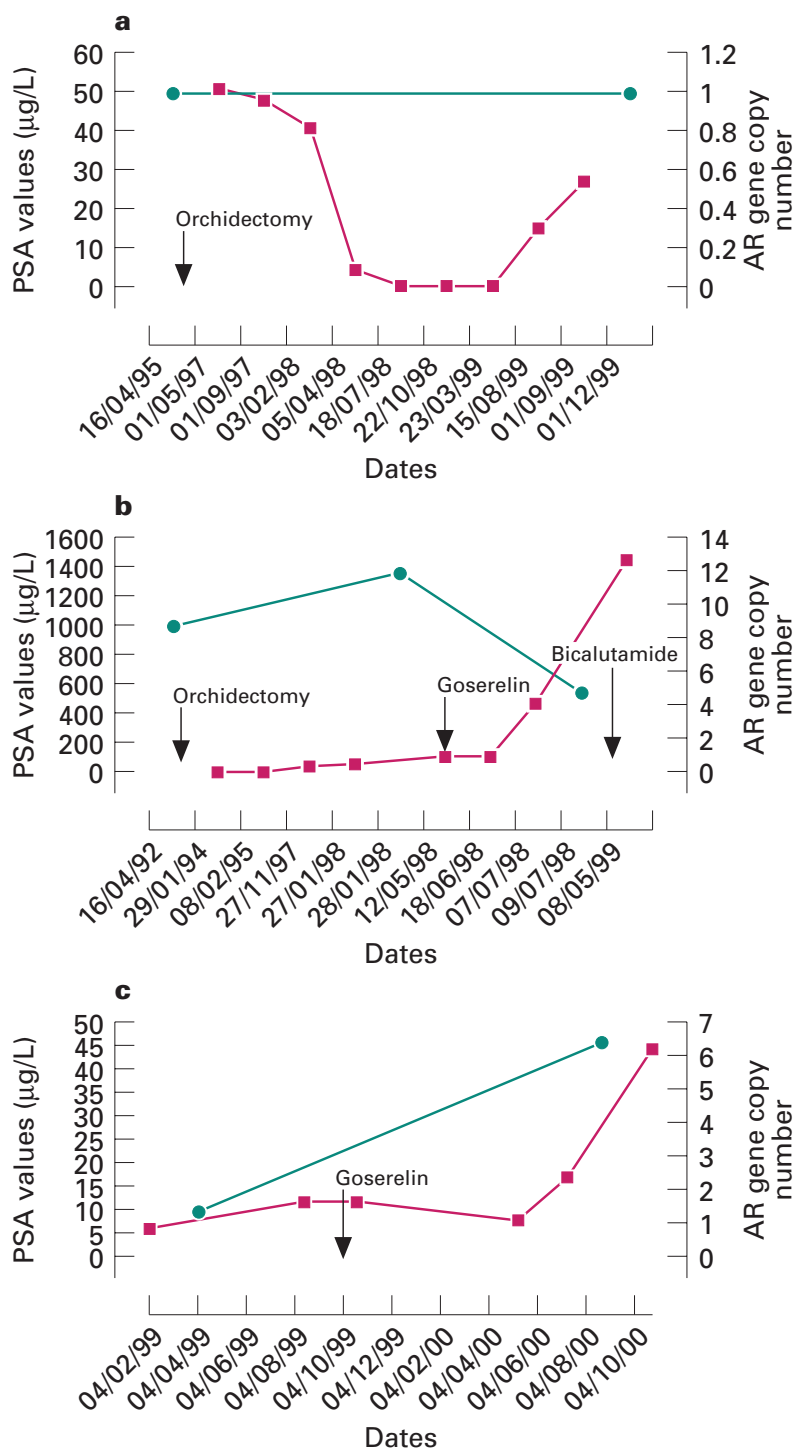

Fig. 1. a, The PSA profile for patient no. 1, showing elevated PSA levels at diagnosis, which reduced in response to treatment and increased when androgen-deprivation therapy failed. This patient, who initially responded to androgen-deprivation therapy, had no abnormalities in biopsies either before or after relapse for $\mathrm{X}$ chromosome or AR copy numbers. $\mathbf{b}$, The PSA profile for patient no. 7 , showing a sharp increase in PSA levels at relapse. There was no available value for PSA at diagnosis, but the records showed that PSA was elevated during this period. This patient responded to androgen-deprivation therapy and had AR amplification in biopsies both before and after relapse. c, The PSA profile for patient no. 20, showing high PSA levels at diagnosis, which increased until treatment was given and then decreased until relapse. This patient responded to androgen-deprivation therapy and had AR amplification only in the biopsy taken after relapse. In all figures the datapoints shown as green circles are the mean AR copy number in each biopsy and those shown as red squares the PSA profile. polysomic for chromosome X. Therefore, $65 \%$ of the recurrent cases investigated had increased AR copy numbers compared with normal prostate gland cells [9]. These findings have since been confirmed by several studies reporting amplification of the AR in $20-30 \%$ and polysomy of chromosome $\mathrm{X}$ in $35-80 \%$ of recurrent tumours $[9,11,12]$. These data have been used to suggest that an increase in AR copy number is involved in the development of hormone-refractory prostate cancer.

In the present study we have, for the first time, investigated AR amplification and copy number in paired tumours before and after hormonal escape in patients with documented initial responses to antiandrogen therapy. The results cast doubt on the role of AR amplification or duplication as mechanisms of hormone escape. Although $65 \%$ of recurrent tumours had an increase in AR copy number, consistent with values previously reported $[9,11,12], 35 \%$ of them also had increased copies of AR in the pretreatment tumours. Despite this, each of these patients had a documented response to antiandrogens (Fig. 1), suggesting that the increased AR copy number did not predict androgen insensitivity. Furthermore, the mean AR copy number after relapse was no higher than that seen in the primary tumours (before therapy). Most strikingly, only three patients had amplification of the AR gene after hormone relapse; of these, one (5\% of primary biopsies) had amplification of the AR gene before antiandrogen therapy. This patient, despite having the greatest amplification found in the study both in tumours before and after relapse, had a full PSA response to antiandrogen therapy. In this patient the PSA relapse could not be related to increased gene amplification of the AR (Fig. 1b).

These results, in which neither increased AR copy number nor amplification preclude a response to antiandrogen therapies, and where relapse is clearly not associated with either increased copy number or amplification of AR, call into question the role of alterations in AR copy number in hormone relapse in prostate cancer.

Although an increase in AR gene copy number does not seem to explain the development of hormonerefractory prostate cancer, we cannot, at present, exclude a change in the level of AR protein expression. Increases in AR expression may lead to hormone resistance and studies are currently underway to address this aspect of AR function in these samples. More recently it has been suggested that the development of hormone-resistant prostate cancer need not involve increased levels of AR but may involve mechanisms which alter AR function with no changes in expression. Recent evidence shows that activation of protein kinase A can up-regulate the expression of androgen-regulated genes via the 
transcription factors cyclic AMP binding protein and activation transcription factor, by androgen-independent activation of $\mathrm{AR}[14,15]$ or via pathways that do not involve AR [16]. The protein kinase C pathway has also been reported to stimulate the expression of androgenregulated genes via $\mathrm{AP}-1$, an $\mathrm{AR}$-independent signal transduction pathway $[16,17]$. Activation of either of these pathways would stimulate prostate tumour growth in the absence of circulating androgens, but whether any of these pathways are involved in androgen escape remains to be determined.

A lack of understanding of the mechanisms driving antiandrogen escape in prostate cancer remains central to the failure to develop viable alternative therapies for patients with hormone-relapsed disease. By investigating paired tumour samples before and after hormone relapse we provide evidence that questions the role of AR amplification in the process of hormone escape. As discussed above, alternative pathways may need to be investigated and could provide novel therapeutic targets for this important disease.

\section{Acknowledgements}

This research was funded by a donation made in memory of Mr Billy Park and by a grant from Astra Zeneca.

\section{References}

1 Goktas S, Ziada A, Crawford ED. Combined androgen blockade for advanced prostatic carcinoma. Prostate Cancer Prostatic Dis 1999; 2: 172-9

2 Maraj BH, Markham AF. Prostate-specific membrane antigen (FOLH1): recent advances in characterising this putative prostate cancer gene. Prostate Cancer Prostatic Dis 1999; 2: 180-5

3 McLeod DG, Crawford ED, DeAntoni EP. Combined androgen blockade: The gold standard for metastatic prostate cancer. Euro Urol 1997; 32 (Suppl. 3): 70-7

4 Gregory CW, Hamil KG, Kim D et al. Androgen receptor expression in androgen independent prostate cancer is associated with increased expression of androgen regulated genes. Cancer Res 1998; 58: 5718-24

5 Kemppainen JA, Lane MA, Sar M, Wilson EM. Androgen receptor phosphorylation, turnover, nuclear transport, and transcriptional activation. J Biol Chem 2000; 267: 968-74

6 Trapman J, Brinkmann AO. The androgen receptor in prostate cancer. Pathology Res Prac 1996; 192: 752-60

7 Giovannucci E, Stampfer MJ, Krithivas K et al. Erratum: The CAG repeat within the androgen receptor gene and its relationship to prostate cancer. Proc Natl Acad Sci USA 1997; 94: 8272-81
8 Newling D, Fossa SD, Andersson L et al. Assessment of hormone refractory prostate cancer. Urology 1997; 49 (Suppl. 4A): 46-53

9 Visakorpi T, Hytinen E, Koivisto P et al. In vivo amplification of the androgen receptor gene and progression of human prostate cancer. Nature Genet 1995; 9: 401-6

10 Koivisto P, Kononen J, Palmberg C et al. Androgen receptor gene amplification: a possible molecular mechanism for androgen deprivation therapy failure in prostate cancer. Cancer Res 1997; 57: 314-9

11 Miyoshi Y, Uemura H, Fujinami K et al. Fluorescence in situ hybridization evaluation of c-myc and androgen receptor gene amplification and chromosomal anomalies in prostate cancer in Japanese patients. Prostate 2000; 43: 225-32

12 Bubendorf L, Kononen J, Koivisto P et al. Survey of gene amplifications during prostate cancer progression by high throughput fluorescence in situ hybridization on tissue microarrays. Cancer Res 1999; 59: 803-6

13 Bartlett JMS, Watters AD, Ballantyne SA, Going JJ, Grigor KM, Cooke TG. Is chromosome 9 loss a marker of disease recurrence in transitional cell carcinoma of the urinary bladder? BJC 1998; 77: 2193-8

14 Fronsdal K, Engedal N, Slagsvold T, Saatcioglu F. CREB binding protein is a co-activator for the androgen receptor and mediates cross-talk with AP-1. J Biol Chem 1998; 273: $31853-9$

15 Sadar MD. Androgen independent induction of prostate specific antigen gene expression via cross talk between the androgen receptor and protein kinase A signal transduction pathways. J Biol Chem 1999; 274: 7777-83

16 Sadar MD, Hussain M, Bruchovsky N. Prostate cancer; molecular biology of early progression to androgen independence. Endocrine Related Cancer 1999; 6: 487-502

17 Sato N, Sadar MD, Bruchovsky N et al. Androgenic induction of prostate-specific antigen gene is repressed by protein-protein interaction between the androgen receptor and AP-1/c-Jun in the human prostate cancer cell line LNCaP. J Biol Chem 1997; 272: 17 485-94

\section{Authors}

J. Edwards, PhD, Research Scientist. N.S. Krishna, FRCS, Research Fellow. R. Mukherjee, MB, ChB, Senior House Officer. A.D. Watters, MSc, Research Scientist. M.A. Underwood, MD, FRCS(Urol), Consultant Urologist. J.M.S. Bartlett, PhD, Senior Lecturer.

Correspondence: J. Edwards, University Department of Surgery, Level II, Queen Elizabeth Building, Glasgow Royal Infirmary, Glasgow G31 2ER, UK.

e-mail: je10b@clinmed.gla.ac.uk

Abbreviation: AR, androgen receptor. 